\title{
APPLETS CON GEOGEBRA EN EL RENDIMIENTO ACADÉMICO EN MATEMÁTICA DE LOS ESTUDIANTES DEL II CICLO ESCUELA PROFESIONAL DE ADMINISTRACIÓN
}

\author{
Applets with Geogebra in the Academic Performance in Mathematics of \\ Students of the II Cycle - Professional School of Administration \\ Pablo Alfredo Sifuentes Damián', Carlos Roberto Pesantes Rojas', Jorge Luis Rojas Paz', \\ Aida Nerida Falcón Cerna', Gabriel Alberto Manes Cangana'.
}

\section{RESUMEN}

La investigación se centró en determinar el efecto que genera el uso de Applets con Geogebra en la Asignatura Matemática Aplicada a los Negocios en el tema de límites de funciones reales de variable real, sobre el Rendimiento Académico de los Estudiantes del II ciclo de la Escuela Profesional de Administración de la Universidad Nacional José Faustino Sánchez Carrión, 2016. El método empleado aplicó una prueba de pre-test y otra de post-test, tanto al grupo experimental (A) como el grupo control (B), desarrollándose las clases en el grupo experimental con el uso del software matemático Geogebra y en el grupo control, mediante una estrategia docente tradicional. Así mismo se utilizó la prueba $U$ de Mann Whitney dado que las muestras de las calificaciones no se distribuyen de forma normal en cada uno de los grupos. En los resultados se obtuvo que la utilización de Applets construidos con el software matemático Geogebra en el tópico de límites de funciones reales de variable real, determinó que existe diferencia significativa entre el grupo experimental (A) y grupo control (B) con un nivel de significancia del $5 \%$, p-valor< Alfa $(0,032<0,05)$. Se concluyo que el uso de Applets construidos en el software matemático Geogebra mejora el Rendimiento Académico en matemática de los estudiantes del Il ciclo de la Escuela Profesional de Administración.

Palabras clave: Software matemático, Applets.

\begin{abstract}
The research focused on determining the effect generated by the use of Applets with Geogebra in the Mathematical Subject Applied to Business in the subject of limits of real functions of real variable, on the Academic Performance of the Students of the II cycle of the Professional School of Administration of the National University José Faustino Sánchez Carrión, 2016. The method used applied a pre-test and a post-test, both to the experimental group (A) and the control group (B), developing the classes in the experimental group with the use of the mathematical software Geogebra and in the control group, by means of a traditional teaching strategy. Likewise, the Mann Whitney $U$ test was used since the samples of the grades are not distributed normally in each of the groups. In the results it was obtained that the use of Applets constructed with the mathematical software Geogebra in the topic of limits of real functions of real variable, determined that there is a significant difference between the experimental group $(A)$ and the control group $(B)$ with a level of significance of $5 \%$, p-value < Alpha $(0.032<0.05)$. It was concluded that the use of Applets built in the mathematical software Geogebra improves the Academic Performance in mathematics of the students of the II cycle of the Professional School of Administration
\end{abstract}

Keywords: Mathematical software, Applets.

${ }^{1}$ Facultad de Ciencias, Universidad Nacional José Faustino Sánchez Carrión. Email: vinvestigacion@unifs.edu.pe 


\section{INTRODUCCIÓN}

.El continuo avance tecnológico en el mundo ha permitido introducir cambios sustanciales en nuestra vida cotidiana, por ejemplo en las formas de realizar nuestras compras y ventas, en las forma de satisfacer nuestra necesidades primarias y secundarias, en la forma de comunicarnos y por supuesto en nuestra vida profesional. Con respecto a esto último nos obliga hoy en día desde la perspectiva de docentes a asumir nuevos roles, a utilizar e introducir la tecnología en el aula de clase incorporándola en nuestros materiales de enseñanza y permitiéndonos innovar e interactuar de una manera más fluida con nuestros estudiantes al desarrollar nuevas experiencias de aprendizaje.

La Organización de las Naciones Unidas para la Educación, la Ciencia y la Cultura (UNESCO, 2013) establece que: la introducción de las TICs en las aulas pone en evidencia la necesidad de una nueva definición de roles, especialmente, para los alumnos y docentes. Los primeros, gracias a estas nuevas herramientas, pueden adquirir mayor autonomía y responsabilidad en el proceso de aprendizaje, lo que obliga al docente a salir de su rol clásico como única fuente de conocimiento. Esto genera incertidumbres, tensiones y temores; realidad que obliga a una readecuación creativa de la institución escolar (Lugo, 2008). (p.16)

En este sentido consideramos que la enseñanza de las matemáticas en la Universidad Nacional José Faustino Sánchez Carrión debe readecuarse y precisamente ese es el propósito que persigue esta investigación al mostrar que la interacción de los estudiantes con la matemática a partir de la creación de Applets en Geogebra- que es un software donde "Sus desarrolladores son Markus Hohenwarter, Michael Borcherds e Yves Kreis. el cual es un software libre, multilingüe y multiplataforma". (Morante y Vallejo, 2011) - los convierte en estudiantes autónomos, creativos, les permite avanzar a su propio ritmo pero para ello es preciso que el docente abandone su rol de trasmisor de conocimientos y ponga en práctica una nueva metodología de aprendizaje basada en la experimentación de las teorías existentes en un mundo que se abre paso a través de la tecnología de la información y comunicación.

Si bien es cierto que unos pocos docentes de nuestra casa superior de estudios han incorporado en su práctica pedagógica las tecnologías de la información y comunicación aun esta es insipiente, observándose a muchos de ellos pertenecientes a las áreas de ciencias que continúan trabajando con la metodología tradicional, preocupados más en la trasmisión de conocimientos o en la parte del cálculo que en áreas como el razonamiento, análisis y construcción o aún más reconstrucción del conocimiento.

Esta investigación más allá de probar que efectivamente la incorporación de la tecnología en el proceso de aprendizaje de la matemática universitaria produce efectos positivos sobre el nivel de rendimiento académico, muestra que es posible construir a partir de los conocimientos teóricos del docente simuladores o Applets en entornos virtuales como los que proporciona
Geogebra, "lo cual pone de manifiesto la importancia de la visualización dentro del ámbito del proceso del aprendizaje de las matemáticas."(Macias, 2007). Así por ejemplo es posible construir Applets en Geogebra que permitan acercar al estudiante visualmente a la definición del límite de funciones reales de variable real los mismos que lo incentivan también a construir sus propios Applets para otras situaciones,que le da autonomía en su aprendizaje y donde muestran su creatividad al realizar nuevas construcciones y mayor facilidad para interpretar gráfica y dinámicamente definiciones involucradas; además es notorio en la mayoría de los casos su entusiasmo durante el proceso de aprendizaje y el trabajo colaborativo que cuando construyen sus propios conocimientos individual o colectivamente.

\section{MATERIALES Y MÉTODOS}

Para este estudio se utilizaron los laboratorios de cómputo de la Facultad de Ciencias Empresariales en los cuales se instaló el software libre Geogebra disponiendo de 03 horas académicas semanales para su realización, durante el ciclo académico 2016-I.

La poblaciòn de estudio estuvo constituido por los estudiantes matriculados durante el ciclo 2016-I distribuidos en dos secciones $A$ y $B$ por su director de escuela, pertenecientes al segundo ciclo de estudios de la Escuela Profesional de Administración de la Universidad Nacional "José Faustino Sánchez Carrión” Huacho.

Para determinar el rendimiento académico en el tópico de límites de funciones reales de variable real, se aplicó una prueba antes del experimento o pre-test y posteriormente un post-test. El pre-test se caracterizó por tener un dominio conceptual, procedimental y actitudinal con relación al conocimiento previo que traían los estudiantes del primer ciclo de estudios en lo que respecta a ecuaciones, inecuaciones y relaciones y funciones. Para el pos- test se evaluó a los grupos de control y experimental en los dominós conceptual, procedimental y actitudinal en lo referente al tópico de límites de funciones reales de variable real tomando como resultados los promedios finales que aparecen en el registro académico, segundo módulo.

Las notas del pre-test se sometieron al análisis con Spss para verificar que los grupos eran homogéneos. Las notas finales, después de aplicar el pos-test se obtuvieron del registro de evaluación del segundo módulo los cuales también se sometieron al análisis estadístico con el paquete Spss versión de prueba descargada de: http://www.ibm.com/pe-es

\section{RESULTADOS}

Los resultados obtenidos en la investigación, derivados del estudio de las notas finales del segundo módulo de los alumnos del grupo control (B) y del grupo experimental $(A)$ son los siguientes:

En primer lugar, se analizó (Tabla 1) la normalidad de las calificaciones mediante el contraste de Shapiro-Wilk que es adecuado cuando las muestras son pequeñas $(n<50)$ y es una alternativa más potente que el test de Kolmogorov-Smirnov. 
Tabla 1. Pruebas de Normalidad

\begin{tabular}{lrrrrrrr}
\hline & \multicolumn{3}{c}{ Kolmogorov-Smirnova } & \multicolumn{3}{c}{ Shapiro-Will } \\
GRUPO & Estadistico & Gl & Sig. & Estadístico & gl & Sig. \\
\hline Grupo A: Experimental & 209 & 26 &, 005 &, 846 & 26 &, 001 \\
Grupo B: Control &, 168 & 30 &, 031 &, 888 & 30 &, 004 \\
\hline
\end{tabular}

Se observa que los p-valores (Sig.) son menores que el nivel de significación 0,05. Concluyendo que las muestras de las calificaciones no se distribuyen de forma normal en cada uno de los grupos experimental y control. Por lo tanto no se puede realizar la prueba t- de Student para muestras independientes y se utiliza la prueba $U$ de Mann Whitney.

Figura 1.Comparación del Rendimiento Académico.

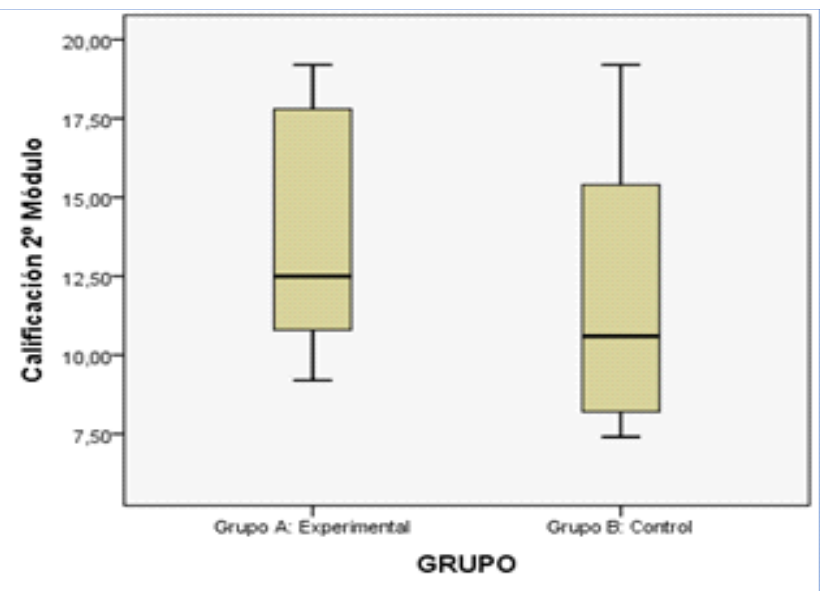

En segundo lugar observando la Figura 1, se puede apreciar que el grupo experimental tiene un mejor rendimiento que el grupo control. Realizando la prueba correspondiente se confirma esto, obteniendo el estadístico siguiente:

Tabla 2: Estadístico de prueba

\begin{tabular}{lr}
\hline ESTADÍSTICO DE PRUEBA* & $\begin{array}{c}\text { Calificación } \\
2^{\circ} \text { Módulo }\end{array}$ \\
\hline U de Mann-Whitney & 259,500 \\
p-valor & 0,032 \\
* Variable de agrupación: GRUPOS (A, B) \\
\hline
\end{tabular}

Como el p-valor $<$ Alfa $(0,032<0,05)$, se puede concluir que existe suficiente evidencia estadística a un nivel de significancia del $5 \%$ para afirmar que existe diferencia significativa entre el grupo experimental(A) y grupo control (B).

Haciendo un análisis más exhaustivo, al hacer la clasificación por rendimiento académico de acuerdo al reglamento académico, se puede comprobar que efectivamente el grupo A cuyos alumnos recibieron la capacitación del software matemático Geogebra tienen un mejor rendimiento que los del grupo $B$ (control), salvo en el grupo de [14 - 16]. Esto se puede visualizar en el siguiente gráfico donde se muestran calificaciones proporcionadas por el intranet de la UNJFSC-ORA 2016I:

Figura 2.Calificaciones Intranet UNJFSCC.

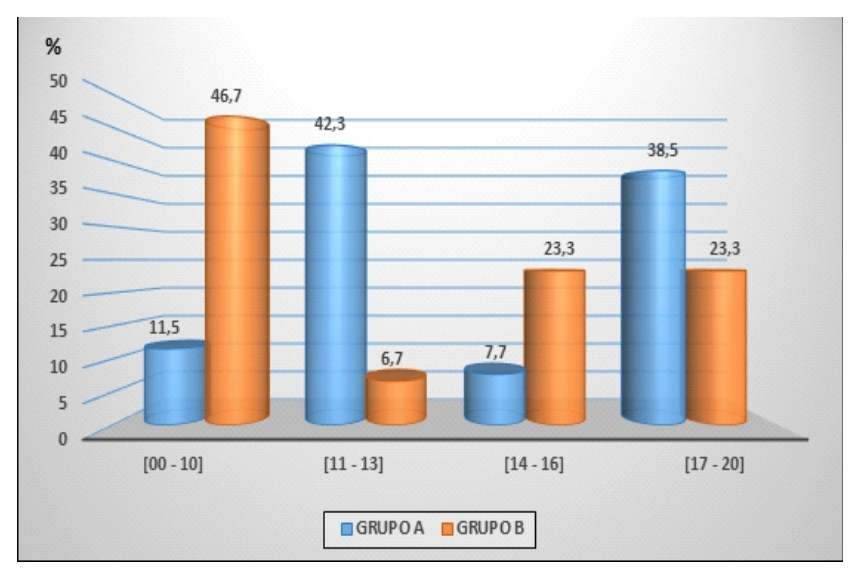

Fuente: Intranet UNJFSC

\section{DISCUSIÓN:}

El propósito del estudio fue determinar el efecto que genera el uso de Applets con Geogebra en la Asignatura de Matemática Aplicada para Negocios en el Rendimiento Académico de los Estudiantes del II ciclo de la Escuela Profesional de Administración de la Universidad Nacional José Faustino Sánchez Carrión.

Los resultados obtenidos indican que existe suficiente evidencia estadística a un nivel de significancia del $5 \%$ para afirmar que existe diferencia significativa entre el grupo experimental(A) y grupo control (B).

En este sentido podemos afirmar que la utilización de Applets en el proceso de aprendizaje de los límites de funciones reales de variable real provoca un efecto beneficioso y significativo en el nivel de rendimiento académico de los estudiantes del II ciclo de la Escuela Profesional de Administración de la Universidad Nacional José Faustino Sánchez Carrión.

Así mismo se ha comprobado que el simulador de Geogebra permite de manera eficiente elaborar Applets relacionados a los contenidos de la asignatura de Matemática Aplicada a los Negocios de la Escuela Profesional de Administración de la Universidad Nacional José Faustino Sánchez Carrión.

\section{AGRADECIMIENTO}

Alos directivos de la Facultad de Ciencias Empresariales de la Universidad Nacional José Faustino Sánchez Carrión por brindar datos y facilidades para la ejecución de la investigación.

\section{REFERENCIA BIBLIOGRÁFICA}

Arya, Lardner\& Ibarra.(2009). Matemáticas Aplicadas a laAdministración y Economía.México: Pearson.

Ariza, A., Linares, S. (2009).Sobre la aplicación y uso del concepto de derivada en el estudio de conceptos económico en estudiantes de bachillerato y universidad. Enseñanza de las Ciencias. 27(1), pp. 121-136. Universidad de Alicante.

Lages, E. (2014). Elementos de Topología General. Instituto de Matemática Pura y Aplicada. Río de 
Janeiro:SBM.

Macías, D. (2007). Las nuevas tecnologías y el aprendizaje de las matemáticas. Recuperado de http://www.rieoei.org/deloslectores/1517Macias.pdf

Mitac, M., Toro, L. (2013). Tópicos de Cálculo. Vol. I Lima:Thales. S.R.L.

Morante, A.; Vallejo, J.A. 2011. "Software libre para el estudio de sistemas dinámicos". La Gaceta de la RSME, 14, pp. 111-132. ISSN.: 1138- 8927.Disponible en:

http://galia.fc.uaslp.mx/ jvallejo/GeogebraMaxima.pdf

Puig, P. (1965). Curso de Geometría Métrica. Madrid: Nuevas Gráficas S.A.
Real, M. (2011).GeoGebra: Una herramienta de software libre con gran potencial en la formación a distancia. Jornadas de Innovación Docente. Universidad de Sevilla.

Spivak, M. (2005). Cálculo Infinitesimal, Barcelona: Reverté.

Unesco. (2013). Enfoques Estratégicos sobre TICS en Educación en América Latina y el Caribe. Recuperado de:http://www.unesco.org/new/fileadmin/MULTIMEDIA /FIELD/Santiago/images/ticsesp.pdf

Kuratowski, K. (1973). Introducción a la teoría de conjuntos y a la topología.Barcelona:Vicens-Vives. 